Chapter 19

\title{
Imaging Patellar Complications After Knee Arthroplasty
}

\author{
Pietro Melloni, Maite Veintemillas, Anna Marin and \\ Rafael Valls
}

Additional information is available at the end of the chapter

http://dx.doi.org/10.5772/53666

\section{Introduction}

Knee arthroplasty, like hip replacement, is becoming increasingly common as the overall population begins to age. The survival rate of the knee implant is also increasing and is now similar to that of hip prostheses (85\%-90\% at 15 years).

Although complications in knee replacements have been widely reported and discussed, the literature contains few studies about patellar complications after total or partial knee arthroplasty.

Patellar complications after knee arthroplasty are infrequent but they can lead to unsatisfactory clinical outcome. Complications are often underestimated because the femoral component makes visualization of these lesions difficult. Evaluation must begin with a thorough history and physical examination. Laboratory tests and imaging studies can provide additional evidence to support a particular diagnosis.

The aim of this chapter is to describe and analyze complications affecting the patella in patients with total or partial knee arthroplasty and to illustrate some representative examples of the spectrum of findings on different imaging techniques, such as plain-film radiography and ultrasound (US), with the emphasis on plain-film findings.

Together with the clinical examination and follow-up, thorough plain-film and computed tomography (CT) studies should be done before and after the surgery. Later follow-up is directed toward identifying complications such as instability/dislocation, fracture, osteonecrosis, infection, erosion, impingement on the prosthesis, patellar or quadriceps tendon tear, and loosening or rupture of the patellar prosthetic button. One large study demonstrated that obtaining plainfilm radiographs immediately after knee arthroplasty is not cost-effective. [1] 
In the follow-up, plain-film radiographs usually suffice for the assessment of patellar complications and are helpful for guiding treatment. Some authors recommend a weight-bearing axial radiograph to better assess patellofemoral kinematics. [2-3] Although radiographs are the mainstay in evaluating loosening or infection, they are limited by their less than optimal sensitivity and specificity. [4]

In one study, the sensitivity and specificity of plain-film radiography compared to the findings at surgery were $77 \%$ and $90 \%$, respectively, for detecting femoral component loosening, and $83 \%$ and $72 \%$, respectively, for detecting tibial component loosening. [5] However, no specific studies about patellar prosthetic button complications are found in the literature.

In the past, the roles of $\mathrm{CT}$ and magnetic resonance imaging (MRI) in the assessment of joint prostheses were inconsequential due to image degradation by artifact. However, improvements in techniques and instrumentation have greatly improved the usefulness of CT and MRI in patients with joint replacements. Although no studies have addressed the routine use of these techniques for the follow-up of asymptomatic patients, some authors recommend CT to look for osteolysis in patients with painful knee prostheses with normal or equivocal radiographs and increased uptake on all three phases of a bone scan. [6] Another group of researchers [7]- [8] recommend multidetector CT in cases where osteolysis is likely, such as those with aseptic loosening and gross polyethylene wear. In patients with loosening, CT examination may also be useful to show the extent and width of lucent zones that may be less apparent on radiographs; in these cases, CT makes it possible to assess rotational alignment of components and to detect subtle or occult periprosthetic fractures of the patella. [9-10]

We use CT to assess component alignment and position as well as rotation of the patella with respect to the femur in patients with knee arthroplasty.

In patients with metallic knee prostheses, we use MRI for very specific indications, such as to evaluate the soft tissues surrounding the patella like the patellar and quadriceps tendons, Hoffa's fat pad, prepatellar subcutaneous tissue, and others. Although MRI is the technique of choice to evaluate the soft tissues [11], its use is seriously limited by drawbacks such as the high cost of acquiring, installing, and maintaining the equipment; magnetic susceptibility; the difficulties of working in a magnetic field; the large number of artifacts; long examination times that may require sedation; discomfort due to the noise inside the scanner; and possible claustrophobia. However, now nearly all implants are non-magnetizable and modern scanners allow images to be manipulated, so magnetic artifacts are no longer a problem. Thus, it could be argued that MRI will eventually supplant US; [12]; for example, MR may be helpful in detecting extracapsular spread of infection and abscess formation. [13]

\section{Material and methods}

Every year between 1998 and 2011, our hospital carried out more than 200 total knee replacements and 10 to 15 implantations of unicompartmental prostheses of the knee. In some knee replacement procedures, the patella was left intact, but in others patellar resurfacing 
was performed or a prosthetic button was implanted. When the patella is intervened, it is often resurfaced with high-density polyethylene, which may be metal backed.

We retrospectively reviewed 1400 consecutive examinations in patients treated with total or partial knee arthroplasty in the last two years; 54 (3.7\%) patients ( 35 women and 19 men) presented patellar complications. Mean patient age was 74 years (range, 55-90 years). In some cases, patients had prostheses in both knees.

All patients were followed up immediately after surgery, at 6 months, and then yearly or when necessary, using anteroposterior, lateral, and axial (Merchant view) radiographs. Lateral and axial projections are better for visualizing and evaluating the evolution of the patella after knee replacement.

In certain cases according to the clinical symptoms, patients underwent US, especially to evaluate the morphological integrity of the patellar and quadriceps tendons and other softtissue structures around the patella.

\section{Results}

The patellar complications that we observed following total knee arthroplasty include instability/dislocation, fracture, osteonecrosis, infection, erosion, impingement on the prosthesis, patellar or quadriceps tendon tear, and loosening or rupture of the patellar prosthetic button. The mean interval from total knee replacement to patellar complication was 5 years and 9 months (range, 5 months- 15 years).

\subsection{Instability/dislocation $(\mathrm{n}=\mathbf{2 1})$}

Patellar instability $(\mathrm{n}=15)$ is the commonest complication after knee arthroplasty. In total knee arthroplasty, most complications related to the extensor mechanism are caused by patellar maltracking instability. [14] Patellar maltracking may result from component malpositioning and limb malalignment, excessive femoral component size, prosthetic design, inadequate patellar resection, or soft-tissue imbalance. [15] Patellofemoral instability likely results most frequently from internal malrotation of the femoral or tibial components. [16]

Malpositioning of femoral and tibial components may affect patellar alignment. Although the axial rotation of the femoral component can be determined using plain-film radiographs or MRI, CT is most commonly used for this purpose. [17] Excessive combined internal rotation of tibial and femoral components is associated with patellar complications. [18] Furthermore, one study [19] found the amount of excessive combined internal rotation was directly proportional to the severity of patellofemoral complications. The rotation of the femoral component can be assessed with relation to the transepicondylar axis, the Whiteside line, or the posterior femoral condyles. The femoral component should be parallel to the transepicondylar axis and the tibial component should be in about 18 degrees of internal rotation with relation to the tibial tubercle. 
Careful radiographic follow-up should be considered when deep flexion is achieved in a knee with a patella baja after total knee arthroplasty (Figure 1). Patellar dislocation $(n=6)$ is mainly due to direct trauma to the patella or to extensor mechanism rupture [20] (Figure 2).

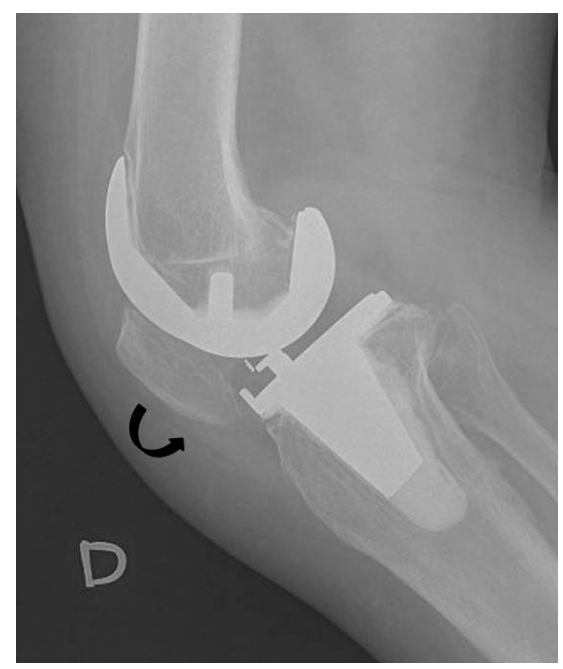

Figure 1. Patellar Instability. A 60-year-old man, five years after total knee replacement. Lateral radiograph reveals caudal displacement of the patella (curved arrow).

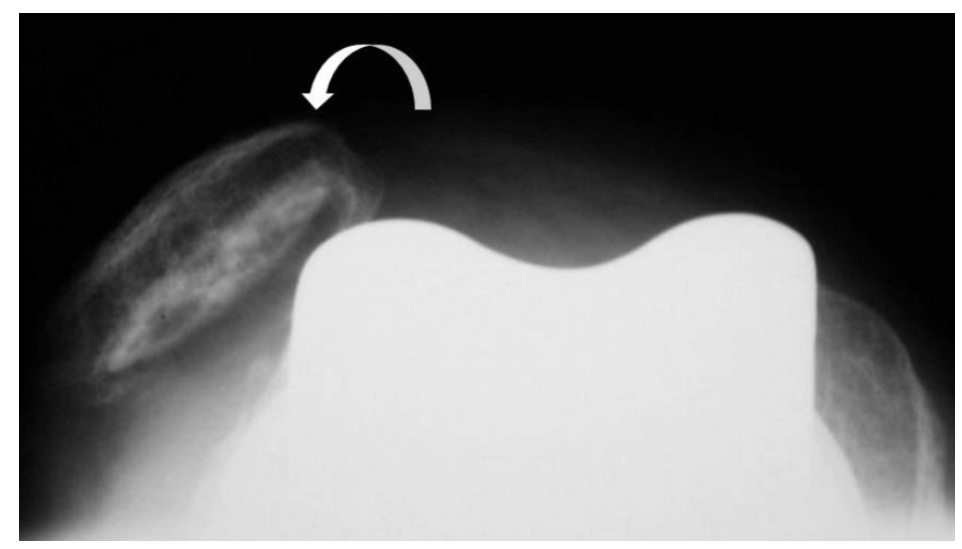

Figure 2. Patellar Dislocation. A 71-year-old woman, five years after total knee replacement. Axial radiograph (Merchant view) of the knee prosthesis with cemented prosthetic button of the patella demonstrates lateral patellar displacement on flexion (curved arrow).

Alterations in the patellotibial distance can occur during total knee arthroplasty due to excessive soft-tissue release that requires elevation of the joint to regain stability and place- 
ment of the polyethylene patellar component distally on the patella. Another cause of acquired patella baja seen commonly in total knee arthroplasty is elevation of the joint line, referred to as pseudo-patella baja. [21]

Radiographic evaluation of the patella primarily uses the lateral view and the sunrise or Merchant's view. This projection should show the central ridge of the patella lying at or medial to the bisector of the trochlear angle. This approach is also helpful for evaluating patellar tilt, but not it is very sensitive for determining the cause of patellofemoral pain.

The lateral view reveals the patellar thickness, inferior or superior positioning, as well as adequate fixation and position of the components. The positioning of the patellar component (centralized or tilted in relation to the trochlear sulcus or subluxated/dislocated) is clearly seen and may reveal the cause of instability. Tilt can be defined as medial or lateral, depending on its relation to the femoral condyles. Subluxation can be measured as displacement from the center of the prosthetic femoral intercondylar groove. [22]

\subsection{Osteonecrosis $(n=5)$}

Patellar resurfacing during total knee arthroplasty remains controversial. Several patellar complications such as fracture, avascular necrosis, and instability are related to resurfacing. On the other hand, some authors report lower re-operation rates and postoperative pain when the patella is resurfaced. Attention should be directed to the ultimate patellar thickness. Whether or not to resurface should be determined based on the exact initial thickness. A thicker patella is prone to instability, whereas a thinner patella is associated with higher complication rates. Patellar fragmentation and sclerosis of the fragments are presumed to represent osteonecrosis (Figure 3). The osteonecrosis may be due to disruption of the vascular network of the patella during total knee replacement surgery. [23] Medial parapatellar arthrotomy, fat pad removal, and lateral release all contribute to patellar devascularization. Evolutional osteonecrosis may lead to patellar fracture.

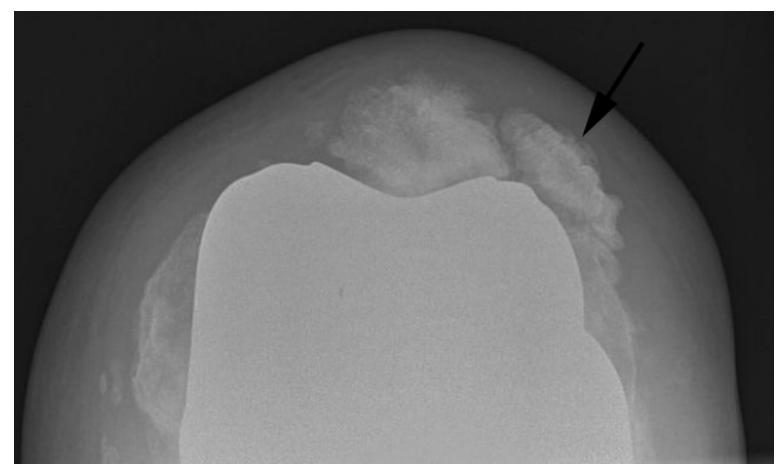

Figure 3. Patellar Necrosis. A 68-year-old man, seven years after total knee replacement. Axial radiograph of the knee prosthesis shows bony sclerosis with fragmentation of the patella (arrow). 


\subsection{Fracture $(n=9)$}

Patellar fractures in association with total knee replacement are uncommon and occur predominantly in patients with resurfaced patellae. [24] Most fractures appear to occur in the first few years after total knee replacement.

Patient, implant, and technical factors are important predisposing causes of these patellar fractures. Avascularity, trauma, fatigue, and stress also play an etiologic role in some patellar fractures.

Trauma to the patella, either direct or indirect, and increased patellofemoral stress are other causes of fracture. Indirect causes might include an eccentric quadriceps muscle contraction associated with a stumble, resulting in an avulsion fracture (Figure 4).

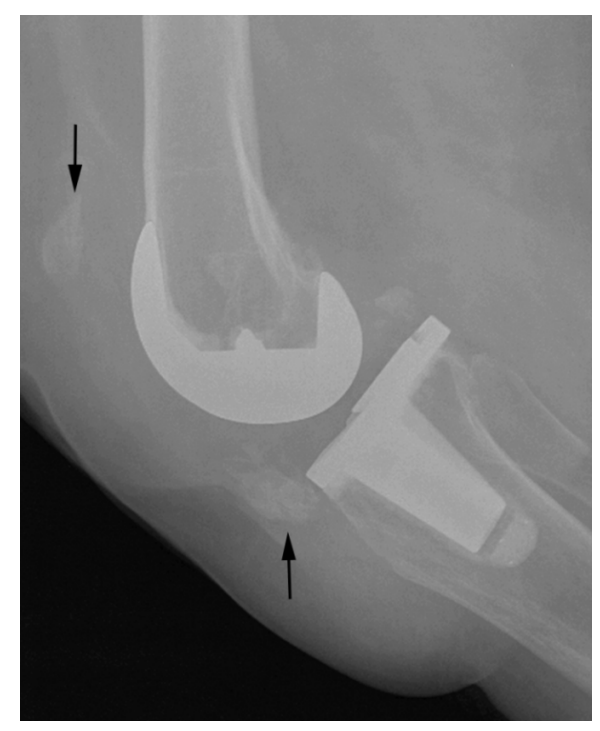

Figure 4. Patellar Avulsion Fracture. A 73-year-old woman, seven years after total knee replacement. Lateral radiograph shows a transverse avulsion fracture in the mid-portion of the patella with displacement of its poles in the cranial-caudal direction (arrows).

Patellar fractures are not associated with prior injury. Because patellar fractures are often asymptomatic and discovered incidentally, follow-up radiographs are essential for their detection. Transverse fractures seem to be related to patellar maltracking, and vertical fractures often occur through a fixation hole. CT or MRI can detect some fractures that go undetected on plain-film radiographs.

Prevention is the best treatment. Important outcome criteria include the integrity of the extensor mechanism, patellar implant fixation, and anatomic location. Surgery on patients with patella fractures has a high complication rate and should be avoided if possible. [25-26] 


\subsection{Infection $(\mathrm{n}=2)$}

Although rare, infection can appear in the patella after total or partial replacement of the knee. [27] Unspecific radiological signs of infection include a lytic lesion or osseous sclerosis in the patella or in the joint facet of the femur in the femoropatellar joint (Figure 5). Clinical symptoms may orient the diagnosis of infection.

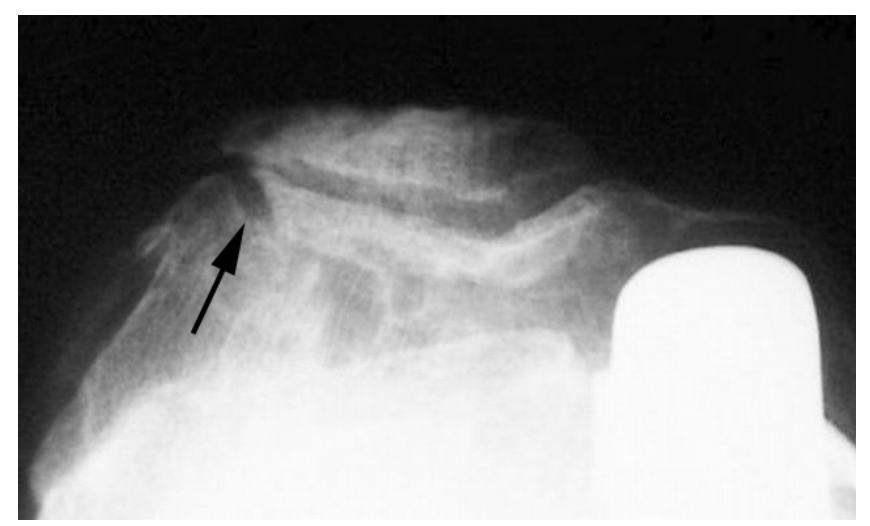

Figure 5. Patellar Infection. A 70-year-old man, nine years after partial knee replacement. Axial radiograph (Merchant view) of the knee prosthesis shows osteolysis on the lateral facet of the femur (arrow) with a non-cemented hemiarthroplasty of the knee, corresponding to a focus of infection, with sclerosis in the patella, suspected of infective infiltration. These findings were confirmed during surgery, and excisional debridement of the infection and total patellectomy were performed. Cultures were positive for Pseudomonas aeruginosa.

Plain-film radiographs are usually negative in the first ten days, even when clinical signs raise suspicion of infection. The radiological presentation varies, sometimes including localized rarefaction in the patella with or without sequestrum, or osseous destruction of the patella with or without an irregular bony fragment adjacent.

Surgical biopsy would provide the definitive diagnosis. The treatment of osteolytic lesions of the patella should be surgical.

\subsection{Erosion/Impingement $(\mathrm{n}=6)$}

Patellar instability can cause erosion $(n=2)$ in the joint facet of the patella due to friction with the femoral component of the knee arthroplasty (Figure 6). The erosion may appear as a lytic lesion that can simulate a subchondral cyst due to any arthritic process or small particle disease. Careful comparison with the pre-arthroplasty plain-films is essential. The erosion should not be confused with a dorsal defect in the posterior surface of the patella that occasionally persists into later life. The dorsal patellar defect is usually well delineated.

Patellar impingement $(n=4)$, the so-called patellar clunk syndrome, results from the formation of a fibrous nodule over the proximal pole of the patella and reportedly occurs in cases 
of total kneed arthroplasty in which a posterior stabilized design is utilized. [28] Arthroscopic or open resection of the fibrous nodule can eliminate this syndrome.

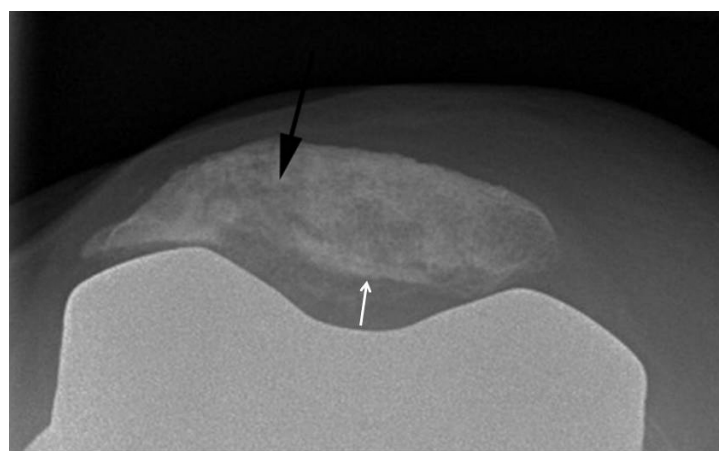

Figure 6. Patellar Osteolysis. A 75-year-old man, three years after total knee replacement. Axial radiograph (Merchant view) shows osteolysis of the lateral facet of the patella (black arrow) due to the loosening of both the total knee prosthesis and the patellar prosthetic button (white arrow). There was clinical suspicion of infection but cultures were negative.

Patellar impingement also is seen when patella baja develops after posterior stabilized total knee arthroplasty and when the patella becomes impinged against the femoral component (Figure 7). [29] Patellofemoral complications (osteoarthritis and impingement) are rarely seen after total replacement and even more rarely after unicompartmental arthroplasty [30], so their long-term consequences are not well known.

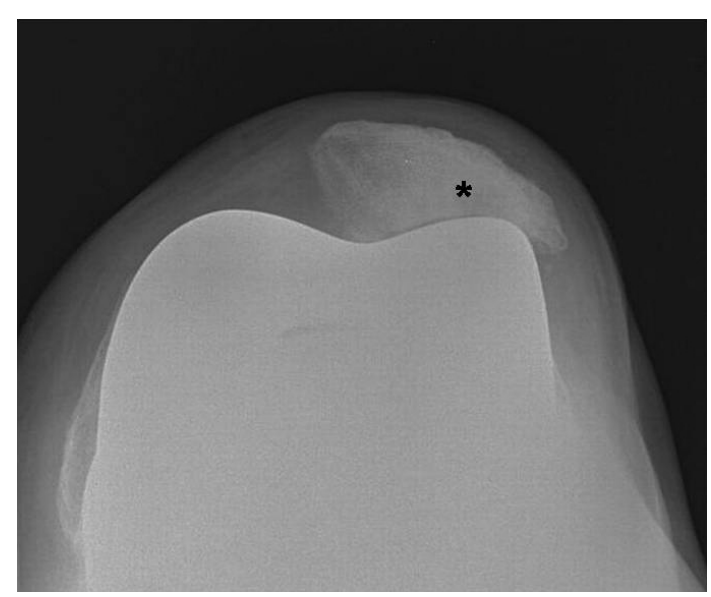

Figure 7. Patellar Impingement. A 71-year-old man, four years after total knee replacement. Axial radiograph (Merchant view) shows a reduction in the space between the knee arthroplasty and the patella, with consequent reactive patellar sclerosis (asterisk). 
However, in our study the symptoms in knees with patellar impingement were usually more severe than in knees with degenerative changes.

\subsection{Loosening or rupture of the patellar prosthetic button $(n=7)$}

A patellar prosthetic button (patellar component) is added to total knee replacement in certain cases. Like all joint prostheses (such as hip, knee, and small joints), the patellar button may loosen or rupture with the same or similar characteristic radiological signs as in the other joints. Loosening of the patellar button (Figure 8) may cause significant anterior pain. Thin fixation pegs, maltracking, and trauma frequently induce component loosening. Revision of a failed patellar component is typically associated with a relatively high complication rate.

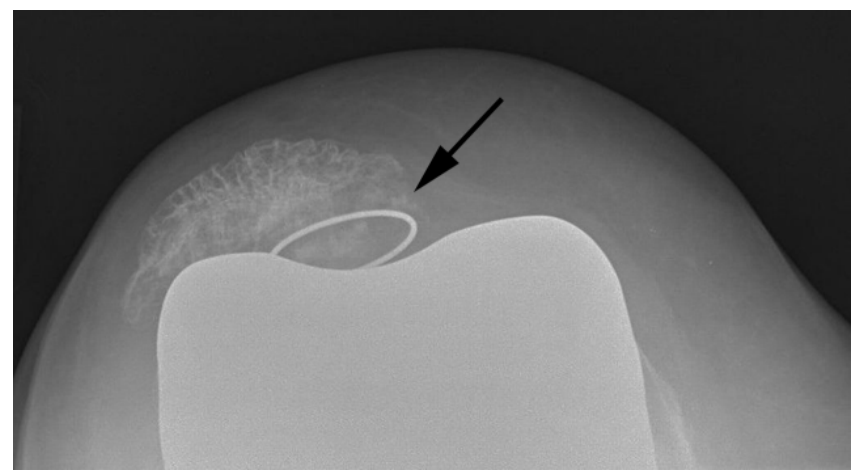

Figure 8. Prosthetic Button Loosening. A 71-year-old woman, two years after total knee replacement. Axial radiograph (Merchant view) shows patellar subluxation with prosthetic button loosening (arrow).

Osseous changes that may be observed in the patellar prosthetic button following total or partial knee arthroplasty include radiolucent lines, osteolysis, change in prosthesis position, and polyethylene wear. Radiolucent lines superimposed on the femoral component can often be obscured by the metal tray if the view is not perfectly tangential to the component surface. Nonprogressive focal radiolucent areas less than $2 \mathrm{~mm}$ in size are often insignificant; however, progressive, circumferential, radiolucent areas larger than $2 \mathrm{~mm}$ are often indicative of prosthesis loosening.

Rupture of the patellar prosthetic button (Figure 9) is rare but can occur due to polyethylene wear, fusion defects in the polyethylene structure [31], or trauma to the patella. [32] The incidence of wear in patients with all-polyethylene and metal-backed components ranges from $5 \%$ to $11 \%$. Congruity, maltracking, and contact force are associated with polyethylene wear. Decreased polyethylene thickness in metal-backed designs is the determining factor for mechanism failure. 


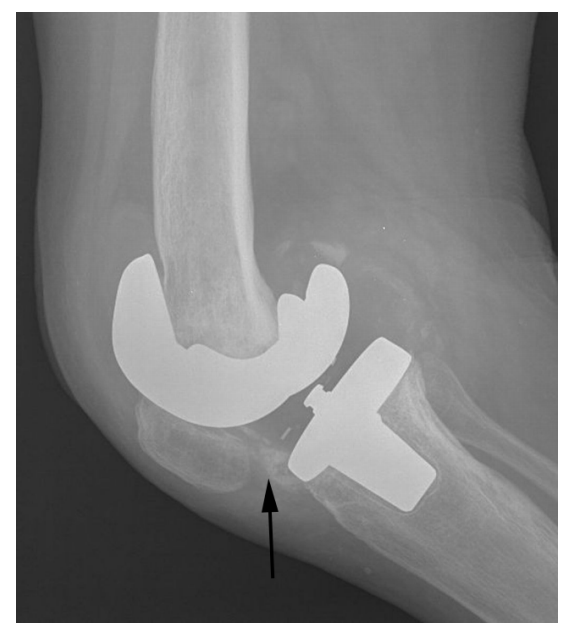

Figure 9. Prosthetic Button Rupture. A 68-year-old woman, four years after total knee replacement. Lateral radiograph shows a rupture of the patellar prosthetic button (arrow) with caudal displacement.

Prosthetic loosening, small particle disease, and infection are the most frequent causes of osteolysis of the patellar component. A change in position of components on serial images is indicative of prosthesis loosening. [33]

\subsection{Patellar or quadriceps tendon tear $(n=4)$}

Rupture of patellar or quadriceps ligaments occurs infrequently. However, the complications of an untreated rupture to the extensor mechanism can be extremely disabling. Contributing factors are excessive dissection and knee manipulation, and trauma. The same mechanical causes that produce patellar fractures can produce patellar [34] or quadriceps [35] tendon tear. US is the method of choice for studying the patellar or quadriceps tendons to confirm or rule out tendon tears (Figures 10 \&11). An abrupt high patella is seen on lateral radiographs in some patients with clinical suspicion of tendon rupture after total knee replacement, but US is necessary to confirm the diagnosis. Although MRI can also be useful in this context, it is not widely used. Other diagnostic possibilities are chronic tendonitis or tendon laxness. Treatment outcomes for ruptured patellar ligaments are not good. 


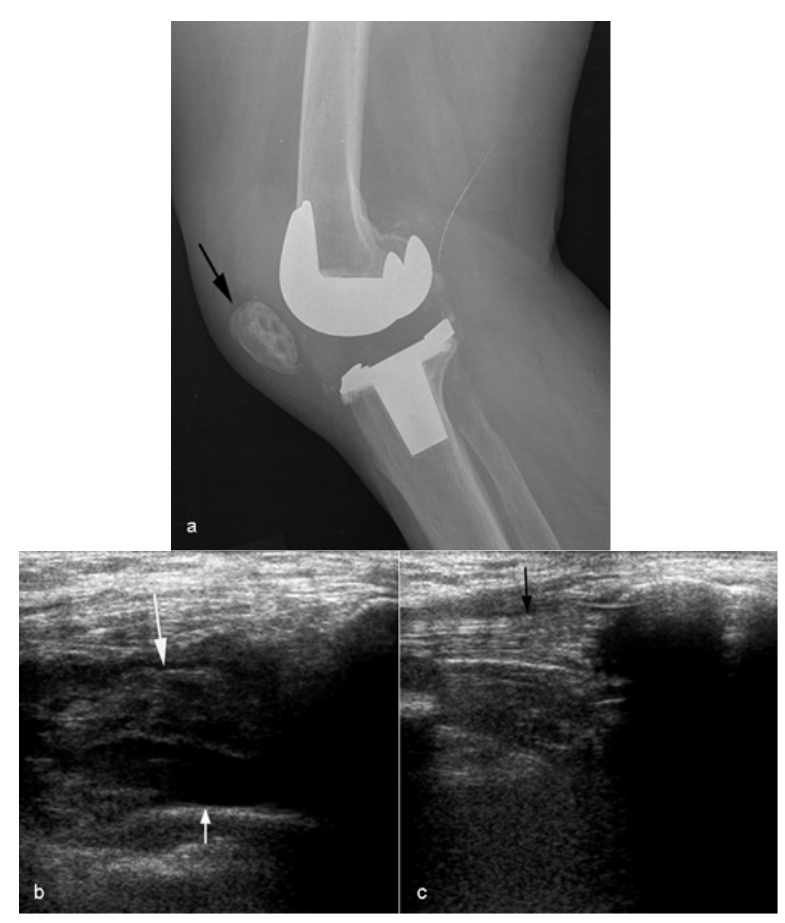

Figure 10. a-c).- QuadricepsTendon Rupture. A 73-year-old woman, ten years after total knee replacement. Lateral radiograph (a) shows patellar displacement and rotation with clinical suspicion of quadriceps tendon tear (black arrow). US (b) confirms a disrupted quadriceps tendon (long white arrow) with a suprapatellar fluid collection (short white arrow) and a $5 \mathrm{~cm}$ gap between the end of the tendon and the patella. Compare with the sonogram of the contralateral knee showing a normal quadriceps tendon (fine black arrow) with total knee replacement (c) in the same patient, who had rheumatoid arthritis.

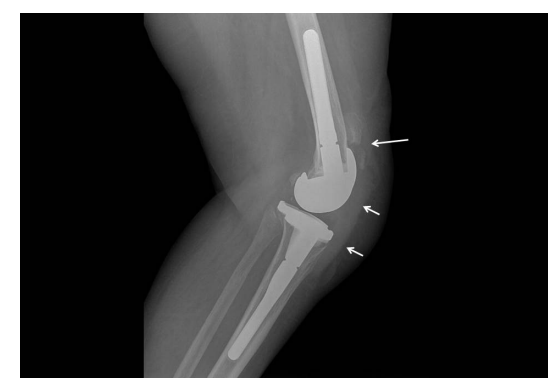

Figure 11. Patellar Tendon Rupture with Patellar Avulsion Fracture. A 69-year-old woman, twelve years after total knee replacement and two years after revision knee replacement with long femoral and tibial stems. Lateral radiograph shows cranial displacement with transverse avulsion fracture in the mid-portion of the patella (long arrow). Note the extensive soft-tissue edema in the patellar area (short arrows), leading to suspected patellar tendon rupture, which was confirmed at ultrasonography (not shown). 


\section{Conclusion}

Patellar complications following knee arthroplasty are generally uncommon but often of potential clinical significance. Plain-film radiographs are essential for the evaluation of patellar complications after surgery and should be the initial imaging study performed. Careful attention to initial prosthesis placement and comparison of follow-up images will allow subtle abnormalities to be detected in patellar complications. US may have a special role in the evaluation of soft-tissue structures around the patella.

\section{Author details}

Pietro Melloni, Maite Veintemillas, Anna Marin and Rafael Valls

UDIAT Diagnostic Center, Corporació Sanitària i Universitària Parc Taulí, Sabadell, Spain

\section{References}

[1] Mendel E. Singer, PhD and Kimberly E. Applegate, MD. Cost-Effectiveness Analysis in Radiology. Departments of Epidemiology and Biostatistics, Metro Health Medical Center (M.E.S.), and the Department of Radiology, Rainbow Babies and Children's Hospital (K.E.A.), Case Western Reserve University School of Medicine, 10900 Euclid Ave, Cleveland, OH 44106 (M.E.S.)

[2] Nobuyuki Yoshino, Nobuyoshi Watanabe, Yukihisa Fukuda, Yoshinobu Watanabe, Shinro Takai. The influence of patellar dislocation on the femoro-tibial loading during total knee arthroplasty. Knee Surgery, Sports Traumatology, Arthroscopy. November 2011, Volume 19, Issue 11, pp 1817-1822.

[3] Shinro Takai, Nobuyuki Yoshino, Nobuyoshi Watanabe and Yukihisa Fukuda. The effect of patellar eversion to the extension and flexion gaps in total knee arthroplasty. J Bone Joint Surg Br 2010 vol. 92-b no. supp i 160-161.

[4] ACR Appropriateness Criteria ${ }^{\circledR}$ imaging after total knee arthroplasty. Weissman BN, Shah N, Daffner RH, Bancroft L, Bennett DL, Blebea JS, Bruno MA, Fries IB, Hayes CW, Kransdorf MJ, Luchs JS, Morrison WB, Palestro CJ, Roberts CC, Stoller DW, Taljanovic MS, Tuite MJ, Ward RJ, Wise JN, Zoga AC, Expert Panel on Musculoskeletal Imaging. ACR Appropriateness Criteria ${ }^{\circledR}$ imaging after total knee arthroplasty. [online publication]. Reston (VA): American College of Radiology (ACR).

[5] Jonathan Baré, MD, Steven J. MacDonald, Robert B. Bourne, MD. Preoperative Evaluations in Revision Total Knee Arthroplasty. Clinical Orthopaedics and Related Research. Number 446, pp. 40-44@ 2006 Lippincott Williams \& Wilkins. 
[6] Use of multi-detector computed tomography for the detection of periprosthetic osteolysis in total knee arthroplasty. Reish TG, Clarke HD, Scuderi GR, Math KR, Scott WN. J Knee Surg. 2006 Oct;19(4):259-64.

[7] Imaging of total knee arthroplasty. Math KR, Zaidi SF, Petchprapa C, Harwin SF. Semin Musculoskelet Radiol. 2006 Mar;10(1):47-63. Review.

[8] Imaging of knee arthroplasty. Miller TT. Eur J Radiol. 2005 May;54(2):164-77. Review.

[9] Can CT-based patient-matched instrumentation achieve consistent rotational alignment in knee arthroplasty? Tibesku CO, Innocenti B, Wong P, Salehi A, Labey L. Arch Orthop Trauma Surg. 2012 Feb;132(2):171-7. Epub 2011 Oct 18.

[10] Rotational positioning of the tibial tray in total knee arthroplasty: a CT evaluation. Berhouet J, Beaufils P, Boisrenoult P, Frasca D, Pujol N. Orthop Traumatol Surg Res. 2011 Nov;97(7):699-704. Epub 2011 Oct 10.

[11] Magnetic resonance imaging of joint arthroplasty. Potter HG, Foo LF. Orthop Clin North Am. 2006 Jul;37(3):361-73, vi-vii. Review.

[12] Magnetic resonance imaging with metal suppression for evaluation of periprosthetic osteolysis after total knee arthroplasty. Vessely MB, Frick MA, Oakes D, Wenger DE, Berry DJ. J Arthroplasty. 2006 Sep;21(6):826-31.

[13] New MR imaging methods for metallic implants in the knee: artifact correction and clinical impact. Chen CA, Chen W, Goodman SB, Hargreaves BA, Koch KM, Lu W, Brau AC, Draper CE, Delp SL, Gold GE. J Magn Reson Imaging. 2011 May;33(5): 1121-7.

[14] Malo M, Vince KG. The unstable patella after total knee arthroplasty: etiology, prevention, and management. J Am Acad Orthop Surg. 2003 Sep-Oct;11(5):364-71.

[15] Robert Wen-Wei Hsu. The management of the patella in total knee arthroplasty. Chang Gung Med J. 2006 September-October. Vol. 29 No. 5.

[16] Efstathios K Motsis, Nikolaos Paschos, Emilios E Pakos, Anastasios D Georgoulis. Review article: Patellar instability after total knee arthroplasty. Journal of Orthopaedic Surgery 2009;17(3):351-7.

[17] Berger RA, Crossett LS, Jacobs JJ, Rubash HE; Malrotation causing patellofemoral complications after total knee arthroplasty. Clinical Orthopedics and Related Research. 1998 Nov;(356):144-53.

[18] Vanbiervliet J, Bellemans J, Innocenti B, et al. The influence of malrotation and femoral component material on patellofemoral wear during gait. Journal Of Bone \& Joint Surgery, British Volume [serial online]. October 2011;93(10):1348-1354.

[19] Blisard R. Component internal rotation malrotation a factor in pain after TKA. Orthopedics Today [serial online]. September 2011;31(9):24.

[20] Rand JA. Extensor mechanism complications following total knee arthroplasty. J Knee Surg 2003;16:224-8. 
[21] Chonko DJ, Lombardi AV Jr, Berend KR. Patella baja and total knee arthroplasty (TKA): etiology, diagnosis, and management. Surg Technol Int. 2004;12:231-8.

[22] Healy WL, Wasilewski SA, Takei R, Oberlander M. Patellofemoral complications following total knee arthroplasty. Correlation with implant design and patient risk factors. J Arthroplasty 1995;10:197-201.

[23] Kyung Ah, Chun Kenjirou, Ohashi D, Lee Bennett, Georges Y, El-Khoury. Patellar Fractures After Total Knee Replacement. AJR AM J Roent. 2005; sept, 185:655-660.

[24] Aglietti P, Baldini A, Buzzi R, Indelli PF. Patella resurfacing in total knee replacement: functional evaluation and complications. J Bone Joint Surg Am. 2002 Jul;84$\mathrm{A}(7): 1132-7$.

[25] Bourne RB. Fractures of the patella after total knee replacement. Orthop Clin North AM. 1999 Apr; 30(2):287-91.

[26] Ortiguera CJ, Berry DJ. Patellar fracture after total knee arthroplasty. J Bone Joint Surg Am. 2002 Apr;84-A(4):532-40.

[27] Keating EM, Haas G, Meding JB. Patella fracture after total knee replacements. Clin Orthop. 2003 Nov;(416):93-7.

[28] Beight JL, Yao B, Hozack WJ, Hearn SL, Booth RE Jr. The patellar "clunk" syndrome after posterior stabilized total knee arthroplasty. Clin Orthop Relat Res. 1994 Feb; (299):139-42.

[29] Maeno S, Kondo M, Niki Y, Matsumoto H. Patellar impingement against the tibial component after total knee arthroplasty. Clin Orthop Relat Res. 2006. Nov; 452: 265.

[30] Matthew B. Collier, MS, et al. Osteolysis After Total Knee Arthroplasty: Influence of Tibial Baseplate Surface Finish and Sterilation of Polyethylene Insert. In The Journal of Bone and Joint Surgery. December 2005. Vol. 87-A. No. 12. Pp. 2702-2708.

[31] Hernigou P, Deschamps G. Patellar impingement following unicompartmental arthroplasty. J Bone Joint Surg Am. 2002 Jul;84-A(7):1132-7.

[32] MacCollum MS 3rd, Karpman RR. Complications of the PCA anatomic patella. Orthopedics. 1989 Nov;12(11):1423-8.

[33] William H Harris. Osteolysis and particle disease in hip replacement. A review. Acta Ortho Scand 1994:65 113-123.

[34] Rand JA, Morrey BF, Bryan RS. Patellar tendon rupture after total knee arthroplasty. Clin Orthop Relat Res. 1989 Jul;(244):233-8.

[35] Dobbs RE, Hanssen AD, Lewallen DG, Pagnano MW. Quadriceps tendon rupture after total knee arthroplasty. Prevalence, complications, and outcomes. Bone Joint Surg Am. 2005 Jan;87(1):37-45. 